\title{
Assessment of epidermal growth factor receptor (EGFR) expression in primary colorectal carcinomas and their related metastases on tissue sections and tissue microarray
}

\author{
Frédéric Bibeau • Florence Boissière-Michot • \\ Jean-Christophe Sabourin • \\ Sophie Gourgou-Bourgade • Michèle Radal • \\ Frédérique Penault-Llorca • Philippe Rochaix • \\ Laurent Arnould • Marie-Pierre Bralet • David Azria • \\ Marc Ychou
}

Received: 3 April 2006 / Accepted: 1 June 2006 / Published online: 25 July 2006

(C) Springer-Verlag 2006

\begin{abstract}
Metastatic colorectal carcinomas (CRC) resistant to chemotherapy may benefit from targeting monoclonal therapy cetuximab when they express the epidermal growth factor receptor (EGFR). Because of its clinical implications,
\end{abstract}

F. Bibeau $(\bowtie) \cdot F$. Boissière-Michot $\cdot$ M. Radal

Department of Pathology, Centre Régional de Lutte Contre le

Cancer Val d'Aurelle,

34298 Montpellier, Cedex 5, France

e-mail: fbibeau@valdorel.fnclcc.fr

S. Gourgou-Bourgade

Department of Biostatistics, Centre Régional de Lutte Contre le

Cancer Val d'Aurelle,

Montpellier, France

J.-C. Sabourin

Department of Pathology, Hôpital Charles Nicolle,

Rouen, France

F. Penault-Llorca

Department of Pathology, Centre Jean Perrin,

Clermont Ferrand, France

P. Rochaix

Department of Pathology, Institut Claudius Regaud,

Toulouse, France

\section{Arnould}

Department of Pathology, Centre Georges-François Leclerc,

Dijon, France

M.-P. Bralet

Department of Pathology, Hôpital Paul Brousse,

Villejuif, France

D. Azria $\cdot$ M. Ychou

Department of Oncology, Centre Régional de Lutte Contre le

Cancer Val d'Aurelle,

Montpellier, France we studied EGFR expression by immunohistochemistry on tissue sections of primary CRC $(n=32)$ and their related metastases $(n=53)$. A tissue microarray (TMA) was generated from the same paraffin blocks to determine whether this technique could be used for EGFR screening in CRC. On tissue sections, $84 \%$ of the primary CRC and 94\% of the metastases were EGFR-positive. When matched, they showed a concordant EGFR-positive status in $78 \%$ of the cases. Moreover, staining intensity and extent of EGFR-positive cells in the primary CRC correlated with those observed in the synchronous metastases. On TMA, $65 \%$ of the primary CRC, $66 \%$ of the metastases, and $43 \%$ of the matched primary CRC metastases were EGFRpositive. There was no concordant EGFR status between the primary and the metastatic sites. A strong discrepancy of EGFR status was noted between TMA and tissue sections. In conclusion, EGFR expression measured in tissue sections from primary $\mathrm{CRC}$ and their related metastases was found to be similar and frequent, but it was significantly underestimated by the TMA technique.

Keywords Epidermal growth factor receptor - Colorectal carcinoma $\cdot$ Metastases $\cdot$ Tissue microarray

\section{Introduction}

Epidermal growth factor receptor (EGFR), also called HER1, is a member of the transmembrane tyrosine kinase receptor family. In normal and malignant cells, the activation of EGFR receptor cascades has multiple consequences, such as cell growth, differentiation, and proliferation [10]. The EGFR signaling pathway may also 
promote malignant transformation, angiogenesis, and metastatic dissemination [10]. To block the activation of this receptor, targeted therapies have been developed, representing a new and promising strategy for cancer management [15]. Thus, antibodies directed against EGFR have recently been integrated into the treatment of metastatic colorectal carcinomas (CRC) resistant to chemotherapy [3, 23]. Such a strategy requires an EGFR immunohistochemical assessment by the pathologist to ensure that the targeted receptor is present in the tumor. Cancer patients included in these protocols are not always treated in the same institute for their primary disease and for their metastatic disease. Moreover, metastases are not always available for histology. It is thus possible that EGFR expression be evaluated in only one of the tumor locations (i.e., primary or metastatic). However, few and controversial data are available concerning the EGFR status in its primary site and the related metastatic sites $[6,11,13,24]$.

The first goal of our study was to analyze whether EFGR expression in colorectal primary tumors and their metastatic sites was similar or not. To answer this question, formalinfixed and paraffin-embedded primary CRC from 32 patients and their related synchronous metastases $(n=45)$ were examined using a standardized EGFR immunohistochemical procedure. Additionally, metachronous metastases $(n=8)$ from four out of 32 patients were also processed for EGFR immunohistochemistry.

The second goal of this study was to evaluate EGFR expression on a tissue microarray (TMA) generated from the same paraffin blocks and to compare these results with those obtained by using the whole tissue sections. This comparison was carried out to determine whether the increasingly used TMA technology is a reliable tool for high-throughput EGFR screening in CRC $[17,26]$.

\section{Materials and methods}

Patient characteristics

Clinical and histopathological characteristics of the patients are reported in Table 1. Thirty-two patients, having undergone surgical resection of both the primary tumor and the corresponding metastatic sites in our institution between 2000 and 2004, were selected from a pathological database of colorectal cancer cases. There were 15 men $(47 \%)$ and 17 women (53\%). Median age at surgery was 60 years (ranging from 45 to 81 years). Twenty-one patients (66\%) had colon cancer and 11 (34\%) had rectal cancer. The most common histological type was adenocarcinoma (91\%), the remaining three cases $(9 \%)$ being mucinous carcinomas. According to the latest TNM classification [28], all the patients had stage-IV disease because of synchronous metastases at the time of diagnosis. Among them, four patients received neoadjuvant radiotherapy $(n=2)$ or radiochemotherapy $(n=2)$ for rectal carcinomas and six others received neoadjuvant chemotherapy before surgical resection of their metastases. Eighty-five paraffin-embedded tumor samples from CRC $(n=32)$ and liver, pulmonary, and ovarian metastases ( $n=51,1$, and 1 , respectively) were available. Synchronous metastases $(n=45)$ were unique in 23 patients and multiple $(n=22)$ in nine patients, consisting of two, three, or four locations in the liver (Table 1). Metachronous metastases $(n=8)$ from four patients were also collected and assessed for EGFR immunohistochemistry. They consisted of five multiple liver metastases for one patient, one single liver metastasis for two patients, and one pulmonary metastasis for one patient. Synchronous and metachronous metastases were obtained by metastasectomy. All resected samples were received fresh, then immediately fixed in $10 \% \mathrm{pH}$ neutral formalin for $48 \mathrm{~h}$ and embedded in paraffin before processing.

\section{EGFR immunohistochemistry}

EGFR immunohistochemistry was carried out on freshly sectioned tissue slides which has been shown to be critical for optimal EGFR immunoreactivity [1]. Immunohistochemistry of the tumors was performed by using the Dako autostainer and the EGFR pharmDx ${ }^{\mathrm{TM}}$ kit (K1494, Dako, Glostrup, Denmark), according to the manufacturer's instructions. Briefly, 4- $\mu \mathrm{m}$-thick sections were mounted on silanized slides and allowed to dry overnight at $37^{\circ} \mathrm{C}$. After deparaffinization and rehydratation, slides were incubated with $3 \%$ hydrogen peroxide for $5 \mathrm{~min}$. After washing with the supplied buffer, tissue sections were covered for $5 \mathrm{~min}$ with a proteinase $\mathrm{K}$ solution. The slides were then incubated for 30 min with the primary mouse anti-EGFR monoclonal antibody (clone 2-18C9), which binds to a formalin-resistant epitope near the ligand-binding site on the extracellular domain of EGFR. After two rinses in buffer, the slides were incubated with a horseradish peroxidase-labeled polymer coupled to secondary antibodies for $30 \mathrm{~min}$. Tissue staining was visualized with diaminobenzidine as substrate-chromogen solution. Slides were counterstained with hematoxylin, dehydrated, and mounted. Negative control sections were processed without the primary antibody but with an irrelevant murine IgG1 supplied with the kit. Negative and positive control cell slides (CAMA-1 and HT29 cell lines, respectively), provided with the EGFR pharmDx ${ }^{\mathrm{TM}}$ kit, were also used to ensure that each assay run was performed appropriately and according to protocol specifications. Furthermore, perineurium and normal mucosae on primary CRC and hepatocytes surrounding the metastases were considered as positive internal controls on tumor slides. 
Table 1 Clinical data and EGFR status among primary CRC and their related metastases

\begin{tabular}{|c|c|c|c|c|c|c|c|c|c|c|c|c|c|c|c|}
\hline \multicolumn{6}{|c|}{ Clinical data } & \multicolumn{10}{|c|}{ EGFR status $^{\mathrm{a}}$ on tissue sections } \\
\hline \multirow[t]{2}{*}{ Case no. } & \multirow[t]{2}{*}{ Sex } & \multirow[t]{2}{*}{ Age (years) } & \multirow[t]{2}{*}{ pTNM } & \multirow[t]{2}{*}{ Location } & \multirow[t]{2}{*}{ Neoadjuvant treatment } & \multirow[t]{2}{*}{ Primary site } & \multicolumn{4}{|c|}{$\begin{array}{l}\text { Synchronous } \\
\text { metastases } \\
\text { (location number) }\end{array}$} & \multicolumn{5}{|c|}{$\begin{array}{l}\text { Metachronous } \\
\text { metastases } \\
\text { (location number) }\end{array}$} \\
\hline & & & & & & & 1 & 2 & 3 & 4 & 1 & 2 & 3 & 4 & 5 \\
\hline 1 & M & 60 & T3N1M1 & $\mathrm{C}$ & & + & + & + & + & + & & & & & \\
\hline 2 & M & 60 & T3N2M1 & $\mathrm{R}$ & RCT & + & + & + & + & & & & & & \\
\hline 3 & M & 66 & T2N0M1 & $\mathrm{R}$ & & + & + & + & + & & & & & & \\
\hline 4 & M & 49 & T3N2M1 & $\mathrm{R}$ & $\mathrm{CT}$ & + & + & + & & & + & + & + & + & + \\
\hline 5 & M & 70 & T3N1M1 & $\mathrm{C}$ & & + & + & + & & & & & & & \\
\hline 6 & $\mathrm{~F}$ & 74 & T3N1M1 & $\mathrm{R}$ & $\mathrm{RCT}$ & + & + & + & & & & & & & \\
\hline 7 & $\mathrm{~F}$ & 59 & T3N2M1 & $\mathrm{R}$ & $\mathrm{CT}$ & + & + & + & & & - & & & & \\
\hline 8 & M & 71 & T3N2M1 & $\mathrm{C}$ & & + & + & + & & & & & & & \\
\hline 9 & $\mathrm{~F}$ & 55 & T3N2M1 & $\mathrm{R}$ & & - & + & - & & & & & & & \\
\hline 10 & $\mathrm{~F}$ & 61 & T4N1M1 & $\mathrm{C}$ & $\mathrm{CT}$ & + & + & & & & + & & & & \\
\hline 11 & $\mathrm{~F}$ & 68 & T3N1M1 & $\mathrm{C}$ & $\mathrm{CT}$ & + & + & & & & + & & & & \\
\hline 12 & M & 75 & T3N1M1 & $\mathrm{R}$ & & + & + & & & & & & & & \\
\hline 13 & M & 72 & T3N1M1 & $\mathrm{C}$ & & + & + & & & & & & & & \\
\hline 14 & M & 54 & T3N0M1 & $\mathrm{C}$ & & + & + & & & & & & & & \\
\hline 15 & $\mathrm{~F}$ & 55 & T4N1M1 & $\mathrm{C}$ & & + & + & & & & & & & & \\
\hline 16 & $\mathrm{~F}$ & 57 & T4N2M1 & $\mathrm{C}$ & & + & + & & & & & & & & \\
\hline 17 & $\mathrm{~F}$ & 52 & T3N1M1 & $\mathrm{R}$ & & + & + & & & & & & & & \\
\hline 18 & M & 53 & T3N0M1 & $\mathrm{C}$ & & + & + & & & & & & & & \\
\hline 19 & M & 62 & T4N2M1 & $\mathrm{C}$ & & + & + & & & & & & & & \\
\hline 20 & $\mathrm{~F}$ & 77 & T3N2M1 & $\mathrm{C}$ & & + & + & & & & & & & & \\
\hline 21 & $\mathrm{~F}$ & 49 & T3N1M1 & $\mathrm{C}$ & & + & + & & & & & & & & \\
\hline 22 & $\mathrm{~F}$ & 51 & T3N2M1 & $\mathrm{C}$ & & + & + & & & & & & & & \\
\hline 23 & M & 62 & T3N2M1 & $\mathrm{R}$ & RT & + & + & & & & & & & & \\
\hline 24 & $\mathrm{~F}$ & 65 & T3N1M1 & $\mathrm{C}$ & $\mathrm{CT}$ & + & + & & & & & & & & \\
\hline 25 & M & 56 & T4N1M1 & $\mathrm{C}$ & $\mathrm{CT}$ & + & + & & & & & & & & \\
\hline 26 & $\mathrm{~F}$ & 47 & T3N1M1 & $\mathrm{R}$ & RT & + & + & & & & & & & & \\
\hline 27 & $\mathrm{~F}$ & 59 & T3N1M1 & $\mathrm{C}$ & & + & - & & & & & & & & \\
\hline 28 & $\mathrm{~F}$ & 45 & T3N2M1 & $\mathrm{C}$ & & + & - & & & & & & & & \\
\hline 29 & $\mathrm{~F}$ & 81 & T3N1M1 & $\mathrm{R}$ & & - & + & & & & & & & & \\
\hline 30 & M & 58 & T2N0M1 & $\mathrm{C}$ & & - & + & & & & & & & & \\
\hline 31 & M & 69 & T3N1M1 & $\mathrm{C}$ & & - & + & & & & & & & & \\
\hline 32 & $\mathrm{~F}$ & 68 & T3N2M1 & $\mathrm{C}$ & & - & + & & & & & & & & \\
\hline
\end{tabular}

Liver metastases in all cases except for a metachronous pulmonary metastasis and a synchronous ovarian metastasis in patients 7 and 15 , respectively

$C$ colon, $R$ rectum, $R C T$ radio-chemotherapy, $C T$ chemotherapy before surgical resection of metastasis, $R T$ radiotherapy

${ }^{a}$ Positive if $>1 \%$ of tumor cells expressed EGFR

\section{Tissue microarray}

Tissue blocks appearing to have enough material upon gross inspection were initially selected and hematoxylineosin-stained sections were evaluated by two observers (F. Bibeau and F. Boissière-Michot) for the presence of carcinoma. The areas to be used for the construction of the TMAs were marked on the slide and the donor block. Particular attention was made to select invasive cells near the tumor front. The tissues corresponding to selected areas were sampled using a manual arraying instrument (Manual Tissue Arrayer 1, Beecher Instruments, Sun Prairie, WI, USA). Two TMAs were constructed using $0.6-\mathrm{mm}$ tissue cores. The sampling consisted of three malignant cores from different areas of the tumor from a single case of invasive colorectal cancer, placed at specified coordinates. When possible, normal mucosa was also sampled as internal control. After the arraying 
was completed, TMA blocks were sectioned at a thickness of $4 \mu \mathrm{m}$. One section was stained with hematoxylin-eosin, and EGFR immunostaining using the immunohistochemical system kit EGFR pharmDx ${ }^{\mathrm{TM}}$ was performed on the adjacent section. Among the 32 cases sampled, 31 primary CRC, 29 metastatic sites, and 28 matched sites were assessable with this technology.

\section{EGFR immunohistochemical staining scoring system}

EGFR assessment was realized according to the EGFR pharmDx ${ }^{\mathrm{TM}}$ scoring guidelines. EGFR expression was evaluated on sections including the deepest region of tumor invasion as this region was shown to contain the greatest density of EGFR-positive cells [6]. Results were reported as positive when a complete or incomplete circumferential membrane staining was observed in at least $1 \%$ of the tumor cells. Staining was defined as immunostaining of tumor cell membranes above background level and scored as follows: $1+=$ weak, $2+=$ moderate, and $3+=$ strong. The absence of membrane staining or cytoplasmic staining was reported as negative. The percentage of stained cells was assessed as follows: $1-10,10-50$, and $>50 \%$. Slides were scored by two pathologists (F. Bibeau and J.C. Sabourin), who were blinded to the patients' characteristics. In the case of disagreement (three out of 91 specimens), the EGFR status was determined by consensus after simultaneous dual reexamination.

In addition to these standardized criteria, a semiquantitative analysis was performed taking into account both the staining intensity and the percentage of positive tumor cells.

\section{Statistical analysis}

The cut-off value for negative/positive expression of EGFR was $1 \%$ of tumor cells displaying a membranous staining, as specified by the EGFR pharmDx ${ }^{\mathrm{TM}}$ scoring guidelines. Data were summarized by frequency and percentage for categorical variables and by means, standard deviations, median, and range for continuous variables. For TMA analysis, the percentage of positive cells was calculated from the mean of triple cores.

The correlation between the percentage of positive cells observed on primary tumors and matched metastases was evaluated with the Spearman correlation coefficient. Associations between categorical variables were examined using McNemar's chi2 test. A $P$ value of less than 0.05 was considered statistically significant. Due to the small number of patients with metachronous metastases, only the correlation between the matched primary $\mathrm{CRC}$ and the synchronous metastatic sites was made.

\section{Results}

EGFR expression of primary CRC and their related metastases on tissue sections

EGFR reactivity was not homogeneous throughout the tumors. EGFR immunostaining was mainly observed in the deepest region of the primary CRC or at the periphery of metastases, especially in the liver. Of note, EGFR immunoreactivity was often greater in isolated tumor cells and in small clusters of tumor cells.

Eighty-four percent of primary CRC (27 out of 32) and $94 \%$ of the synchronous metastases (30 out of 32 ) displayed EGFR reactivity in more than $1 \%$ of the tumor cells (Table 1). Overall, all patients displayed an EGFRpositive status considering EGFR-expression in the primary tumor and/or the metastatic sites. EGFR expression was positive at both sites in most of the cases ( 25 out of 32 ; $78 \%$ ) and rarely in only one site, i.e., primitive (two out of $32 ; 6 \%$ ) or metastastic (five out of $32 ; 16 \%$ ). However, the discordant cases (i.e., positive primary sites with negative metastasis and conversely) were not statistically significant $(P=0.453)$.

Nine (28\%), $10(31 \%)$, and eight $(25 \%)$ of the primary CRC and seven (22\%), 14 (44\%), and nine (28\%) of the synchronous metastases displayed a weak, moderate, and strong positivity, respectively. According to the extent of EGFR immunoreactivity, five (16\%) primary CRC had no reactivity, $12(37.5 \%)$ had $<10 \%$ reactive cells, eight $(25 \%)$ had $>10-50 \%$ positive cells, and seven $(22 \%)$ had $>50 \%$ labeled cells. In the synchronous metastases, two cases $(6 \%)$ were negative, $13(41 \%)$ had $<10 \%$ reactive cells, 11 $(34 \%)$ had $>10-50 \%$ positive cells, and six $(19 \%)$ had $>50 \%$ labeled cells.

Interestingly, the percentage of EGFR-positive tumor cells in the primary site was significantly correlated with that observed in the synchronous metastases $\left(r^{2}=0.616\right.$, $P=0.0002)$. This correlation was also demonstrated when combining EGFR scoring (labeling intensity) with the percentage of EGFR-positive tumor cells $\left(r^{2}=0.559\right.$, $P=0.0009$ ). One example of the pattern of EGFR labeling in both sites is shown in Fig. 1. EGFR expression was positive among the multiple synchronous liver metastases in eight out of nine patients (i.e., 21 out of 22 samples, Table 1). The related primary site of the discordant case (two synchronous metastases: one was EGFR-positive, the other was EGFR-negative) displayed an EGFR-negative status (case number 9, Table 1).

Regarding the analysis of EGFR expression in metachronous metastases, we observed an analogous EGFRpositive status between all the available liver metastases (i.e., five metastases for one patient, one metastasis for two patients) and their related primary sites and synchronous 
Fig. 1 Example of EGFR expression in tissue sections in a primary colorectal carcinoma (a) and the matched metastasis (b). Strong membranous staining of numerous cells in both sites (immunoperoxidase; bar $50 \mu \mathrm{m}$ )
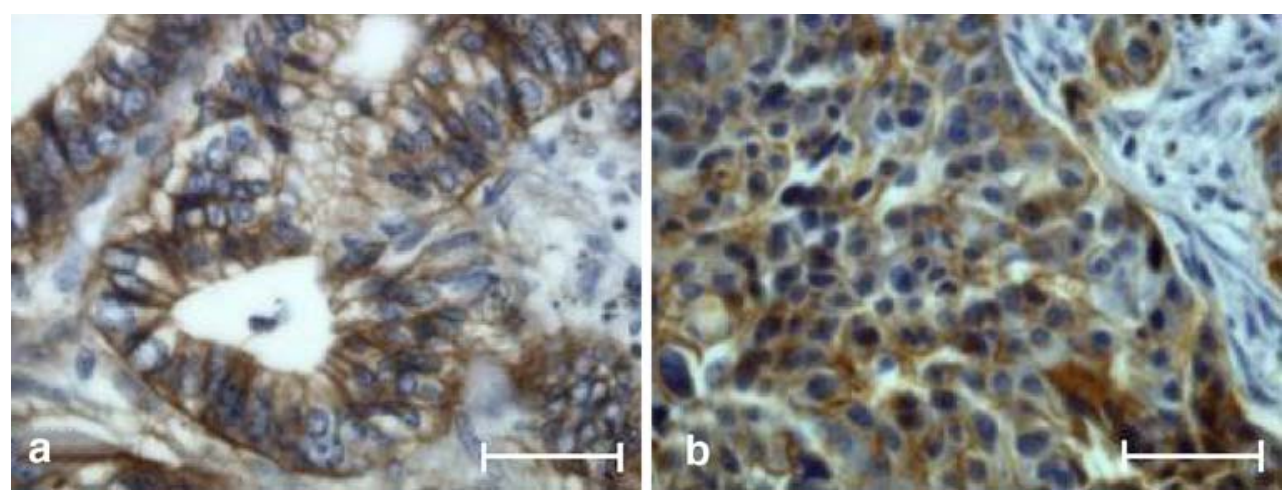

metastases (case numbers 4, 10, and 11, Table 1). The only metachronous pulmonary metastasis available for analysis did not show any EGFR immunoreactivity, whereas both the corresponding hepatic synchronous metastases and the primary site expressed EGFR (case number 7, Table 1).

EGFR expression of primary $\mathrm{CRC}$ and their related metastases on TMA

On the first TMA block, the EGFR status was found to be positive in 18 out of the $31(58 \%)$ assessable primary tumors and 18 out of 29 metastases (62\%) (Table 2). Only nine out of $28(32 \%)$ assessable matched tumors were positive for both the primary and the metastatic sites, whereas four out of $28(14 \%)$ were negative. Fifteen out of 28 (54\%) cases were discordant (i.e., seven positive primary and negative metastases, and eight, conversely).

On the second TMA block, 14 out of $31(45 \%)$ primary tumors and 16 out of $29(55 \%)$ metastases were EGFRpositive (Table 2). Eight out of 28 (29\%) assessable matched tumors were positive for both sites, whereas nine (32\%) were negative. Eleven out of 28 cases $(39 \%)$ were discordant (i.e., four positive primary sites and negative metastases, and seven, conversely).

When combining the two TMAs (six tissue cores), the number of positive cases increased, to reach 20 out of 31 of the primary tumors $(65 \%)$ and 19 out of 29 of the metastases (66\%) (Table 2). Twelve out of $28(43 \%)$ assessable matched tumors were positive for both sites, whereas three $(11 \%)$ were negative. Nevertheless, 13 cases

Table 2 Comparison of EGFR expression in primary CRC and their synchronous metastatic sites on whole tissue sections and TMA

\begin{tabular}{llll}
\hline & Primary site & Metastases & Matched sites \\
\hline Tissue sections & $27 / 32(84 \%)$ & $30 / 32(94 \%)$ & $25 / 32(78 \%)$ \\
First TMA & $18 / 31(58 \%)$ & $18 / 29(62 \%)$ & $9 / 28(32 \%)$ \\
Second TMA & $14 / 31(45 \%)$ & $16 / 29(55 \%)$ & $8 / 28(29 \%)$ \\
Both TMAs & $20 / 31(65 \%)$ & $19 / 29(66 \%)$ & $12 / 28(43 \%)$ \\
\hline
\end{tabular}

Data are expressed as the number of positive cases/total number of specimens
(46\%) remained discordant (i.e., six positive primary and negative metastases, and seven, conversely). Moreover, using the TMA technology and whatever the number of cores (three or six), we failed to demonstrate any correlation of EGFR expression between primitive sites and matched metastases.

Comparison of EGFR expression between tissue sections and TMA

The EGFR status on the triple core-tissue array was significantly underestimated at both sites when compared with tissue sections $(P=0.011$ for both primitive and metastatic sites for the first TMA; $P=0.0005$ and $P=0.0023$ for primitive and metastatic sites, respectively, for the second TMA). When combining both TMAs (six tissue cores), although the number of positive cases increased, we still observed a significant discrepancy between both techniques for primitive sites $(P=0.034)$ and metastases $(P=0.0001)$.

\section{Discussion}

EGFR is a $170-\mathrm{kDa}$ transmembrane cell surface receptor encoded by the human HERI gene, which promotes malignant cell proliferation and cancer progression [15]. Interfering with EGFR cell signaling by targeting strategies represents a novel approach to the treatment of solid tumors [15]. Among them, cetuximab (Erbitux $\left.{ }^{\circledR}\right)$, a chimeric monoclonal human-mouse antibody, is used for the treatment of EGFR-expressing metastatic CRC that have progressed following chemotherapy [3, 23]. To identify patients eligible for this treatment, an immunohistochemical evaluation of EGFR expression is required. Although numerous studies have demonstrated EGFR expression in $70-97 \%$ of CRC $[4,6,29]$, only few and controversial data are available on the EGFR status in distant metastases [6, 11, 13, 24]. Consequently, knowledge of EGFR metastastic status could be of potential value for therapeutic decisions, particularly if it differs from the primary tumors. 
To answer the question as to whether EGFR expression is similar in different tumor sites, we compared the EGFR status of a set of primary CRC and their corresponding synchronous distant metastases, using a standardized immunohistochemistry test (EGFR pharmDx ${ }^{\mathrm{TM}}$, Dako). We also analyzed EGFR expression in metachronous metastases, when available, to study possible changes of EGFR expression during the metastatic process of CRC.

On whole tissue sections, our results demonstrated that EGFR expression was frequent and similar in both the primary and the metastatic sites. In our study, immunoreactivity was observed in 84 and $94 \%$ of the primary tumors and the metastases, respectively (Table 1). The 32 matched primary and synchronous metastatic CRC expressed a concordant EGFR status in $78 \%$ of the cases, all immunopositive, and the discordant cases were not statistically significant $(P=0.453)$. Our data are in agreement with three recent studies that showed a high frequency and concordance of EGFR expression in matched primary tumors and distant metastatic lesions of CRC $[8,11,13]$. To our knowledge, only one study reported no correlation of EGFR expression on primary CRC and related metastases [24].

In addition to the concordant EGFR status between primary and metastatic locations, our study showed that both the staining intensity and the percentage of EGFRpositive cells in the primary site were significantly correlated with those observed in synchronous metastases (Fig. 1). Our work also emphasized the EGFR-positive status among multiple synchronous and metachronous metastatic sites in most of the patients (Table 1). Furthermore, we noticed an analogous EGFR-positive status between all metachronous metastases but one, their related synchronous metastases, and their primary CRC (Table 1). Such data, to our knowledge, have never been reported in clinical samples and seem to agree with the preclinical results, suggesting that EGFR expression is required for the tumor to acquire metastatic potential [19, $22,27]$. Moreover, in our cohort, all the patients displayed an EGFR-positive status. This would mean that most of the patients are potentially eligible for therapy with EGFRtargeted monoclonal antibodies, given the results of the immunohistochemistry.

The clinical relevance of EGFR immunohistochemical detection is today a matter of debate because EGFRnegative patients might respond to cetuximab and EGFRpositive patients might not $[2,3,5,23]$. One reason proposed to explain this lack of correlation is a possible difference in the EGFR status between the primary tumor and the metastatic sites [24]. Our results do not support this hypothesis. The lack of response in EGFR-positive patients could more likely be explained by alterations in the critical downstream signals, activated via other receptors or other pathways. It is also plausible that the lack of correlation between EGFR expression and antitumor activity could be due to the potential for cetuximab to induce antibodydependent cell-mediated cytotoxicity and to the absence of discrimination between the high- and low-affinity receptors with the commonly used antibodies $[2,14,16]$. Moreover, negative immunohistochemical results may be linked to the heterogeneity of EGFR expression and could, in part, represent false negative cases that might respond to EGFR-targeted therapy.

The second goal of our study was to perform the same analysis on TMA generated with the same paraffin blocks used for EGFR assessment on the whole tissue sections. TMA has been employed to study the molecular profile of different cancer types and to test hypotheses regarding colorectal carcinogenesis and prognosis [9, 12, 20]. However, EGFR expression in primary CRC and their related metastases has rarely been studied with this approach and the few published results are controversial $[8,25]$. In our work, TMA was carried on multiple (three) cores, selected from the deepest region of tumor invasion. Nevertheless, the results obtained were systematically lower than those observed on the whole tissue sections. Even when combining the two TMAs, EGFR positivity only reached 65 and $66 \%$ in primary CRC and their related metastases, respectively (Table 2 ). In contrast to what we observed on tissue sections, we failed to demonstrate a correlation of EGFR expression between both sites using the TMA technology. The discordant results between the two technologies may be explained by cases containing rare stained cells (i.e., $<10 \%$ ) or small invasive clusters, which may be not selected when TMA is performed even using three or six cores. These small tumor clusters, also called tumor "budding," belong to a phenomenon which might be involved in metastatic spreading and seem to represent a poor prognostic factor $[18,21,30]$. Such data are of importance because TMA has been recognized as a useful and powerful tool for screening immunohistochemical markers for prognostic purposes and for carcinogenesis studies, as well.

In conclusion, we demonstrated that EGFR expression on whole tissue sections is frequent and similar in primary $\mathrm{CRC}$ and their related metastases. Our results also indicated that the EGFR status of the primary is concordant with those of metastases, whatever the number of sites and the time of occurrence, i.e., synchronous or metachronous. This may have clinical implications because patients are not always treated in the same institute for their primary disease and for their metastatic disease. In our experience, TMA does not represent an appropriate technique for EGFR screening in CRC because it significantly underestimates EGFR expression and does not provide data similar to those obtained with whole tissue sections. Undoubtedly, this technology is a reliable tool for the high-throughput 
assessment of homogeneously distributed proteins [7], but it appears to generate unreliable information with very heterogeneous markers such as EGFR.

Acknowledgement We thank Dr. S.L. Salhi for presubmission editorial assistance.

\section{References}

1. Atkins D, Reiffen KA, Tegtmeier CL, Winther H, Bonato MS, Storkel S (2004) Immunohistochemical detection of EGFR in paraffin-embedded tumor tissues: variation in staining intensity due to choice of fixative and storage time of tissue sections. J Histochem Cytochem 52:893-901

2. Chung KY, Shia J, Kemeny NE, Shah M, Schwartz GK, Tse A, Hamilton A, Pan D, Schrag D, Schwartz L, Klimstra DS, Fridman D, Kelsen DP, Saltz LB (2005) Cetuximab shows activity in colorectal cancer patients with tumors that do not express the epidermal growth factor receptor by immunohistochemistry. J Clin Oncol 23:1803-1810

3. Cunningham D, Humblet Y, Siena S, Khayat D, Bleiberg H, Santoro A, Bets D, Mueser M, Harstrick A, Verslype C, Chau I, Van CE (2004) Cetuximab monotherapy and cetuximab plus irinotecan in irinotecan-refractory metastatic colorectal cancer. N Engl J Med 351:337-345

4. Cunningham MP, Essapen S, Thomas H, Green M, Lovell DP, Topham C, Marks C, Modjtahedi H (2005) Coexpression, prognostic significance and predictive value of EGFR, EGFRvIII and phosphorylated EGFR in colorectal cancer. Int $\mathrm{J}$ Oncol 27:317-325

5. Dei Tos AP, Ellis I (2005) Assessing epidermal growth factor receptor expression in tumours: what is the value of current test methods? Eur J Cancer 41:1383-1392

6. Goldstein NS, Armin M (2001) Epidermal growth factor receptor immunohistochemical reactivity in patients with American Joint Committee on Cancer Stage IV colon adenocarcinoma: implications for a standardized scoring system. Cancer 92:1331-1346

7. Gomaa W, Ke Y, Fujii H, Helliwell T (2005) Tissue microarray of head and neck squamous carcinoma: validation of the methodology for the study of cutaneous fatty acid-binding protein, vascular endothelial growth factor, involucrin and Ki-67. Virchows Arch 447:701-709

8. Gupta M, Varma V, Costonis G, Lawson D, Cohen C (2005) Epidermal Growth Factor Receptor (EGFR) in primary and metastatic colorectal carcinoma. Mod Pathol 18(Suppl 1):105A

9. Hernandez BY, Frierson HF, Moskaluk CA, Li YJ, Clegg L, Cote TR, McCusker ME, Hankey BF, Edwards BK, Goodman MT (2005) CK20 and CK7 protein expression in colorectal cancer: demonstration of the utility of a population-based tissue microarray. Hum Pathol 36:275-281

10. Holbro T, Civenni G, Hynes NE (2003) The ErbB receptors and their role in cancer progression. Exp Cell Res 284:99-110

11. Italiano A, Saint-Paul MC, Caroli-Bosc FX, Francois E, Bourgeon A, Benchimol D, Gugenheim J, Michiels JF (2005) Epidermal growth factor receptor (EGFR) status in primary colorectal tumors correlates with EGFR expression in related metastatic sites: biological and clinical implications. Ann Oncol 16:1503-1507

12. Jourdan F, Sebbagh N, Comperat E, Mourra N, Flahault A, Olschwang S, Duval A, Hamelin R, Flejou JF (2003) Tissue microarray technology: validation in colorectal carcinoma and analysis of p53, hMLH1, and hMSH2 immunohistochemical expression. Virchows Arch 443:115-121
13. Khalifa MA, Rowsell CH, Gladdy RA, Ko YJ, Hanna S, Smith A, Law C (2006) Expression of epidermal growth factor receptor in primary colorectal adenocarcinoma predicts expression in recurrent disease. Am J Clin Pathol 125:229-233

14. Lax I, Bellot F, Howk R, Ullrich A, Givol D, Schlessinger J (1989) Functional analysis of the ligand binding site of EGFreceptor utilizing chimeric chicken/human receptor molecules. EMBO J 8:421-427

15. Lockhart C, Berlin JD (2005) The epidermal growth factor receptor as a target for colorectal cancer therapy. Semin Oncol 32:52-60

16. Mattoon DR, Lamothe B, Lax I, Schlessinger J (2004) The docking protein Gab1 is the primary mediator of EGFstimulated activation of the PI-3K/Akt cell survival pathway. BMC Biol 2:24

17. Mobasheri A, Airley R, Foster CS, Schulze-Tanzil G, Shakibaei M (2004) Post-genomic applications of tissue microarrays: basic research, prognostic oncology, clinical genomics and drug discovery. Histol Histopathol 19:325-335

18. Park KJ, Choi HJ, Roh MS, Kwon HC, Kim C (2005) Intensity of tumor budding and its prognostic implications in invasive colon carcinoma. Dis Colon Rectum 48:1597-1602

19. Parker C, Roseman BJ, Bucana CD, Tsan R, Radinsky R (1998) Preferential activation of the epidermal growth factor receptor in human colon carcinoma liver metastases in nude mice. J Histochem Cytochem 46:595-602

20. Prall F, Ostwald C, Nizze H, Barten M (2004) Expression profiling of colorectal carcinomas using tissue microarrays: cell cycle regulatory proteins p21, p27, and p53 as immunohistochemical prognostic markers in univariate and multivariate analysis. Appl Immunohistochem Mol Morphol 12:111-121

21. Prall F, Nizze H, Barten M (2005) Tumour budding as prognostic factor in stage I/II colorectal carcinoma. Histopathology 47:17-24

22. Radinsky R, Risin S, Fan D, Dong Z, Bielenberg D, Bucana CD, Fidler IJ (1995) Level and function of epidermal growth factor receptor predict the metastatic potential of human colon carcinoma cells. Clin Cancer Res 1:19-31

23. Saltz LB, Meropol NJ, Loehrer PJ Sr, Needle MN, Kopit J, Mayer RJ (2004) Phase II trial of cetuximab in patients with refractory colorectal cancer that expresses the epidermal growth factor receptor. J Clin Oncol 22:1201-1208

24. Scartozzi M, Bearzi I, Berardi R, Mandolesi A, Fabris G, Cascinu S (2004) Epidermal growth factor receptor (EGFR) status in primary colorectal tumors does not correlate with EGFR expression in related metastatic sites: implications for treatment with EGFRtargeted monoclonal antibodies. J Clin Oncol 22:4772-4778

25. Shia J, Klimstra DS, Li AR, Qin J, Saltz L, Teruya-Feldstein J, Akram M, Chung KY, Yao D, Paty PB, Gerald W, Chen B (2005) Epidermal growth factor receptor expression and gene amplification in colorectal carcinoma: an immunohistochemical and chromogenic in situ hybridization study. Mod Pathol 18:1350-1356

26. Simon R, Mirlacher M, Sauter G (2004) Tissue microarrays. Biotechniques 36:98-105

27. Singh RK, Tsan R, Radinsky R (1997) Influence of the host microenvironment on the clonal selection of human colon carcinoma cells during primary tumor growth and metastasis. Clin Exp Metastasis 15:140-150

28. Sobin LH, Wittekind CH (eds) (2002) TNM classification of malignant tumours, 6th edn. Wiley-Liss, New York

29. Spano JP, Fagard R, Soria JC, Rixe O, Khayat D, Milano G (2005) Epidermal growth factor receptor signaling in colorectal cancer: preclinical data and therapeutic perspectives. Ann Oncol 16:189-194

30. Ueno H, Murphy J, Jass JR, Mochizuki H, Talbot IC (2002) Tumour 'budding' as an index to estimate the potential of aggressiveness in rectal cancer. Histopathology 40:127-132 